\title{
Cluster expansion Monte Carlo study of phase stability of vanadium nitrides
}

\author{
C. Ravi, * H. K. Sahu, and M. C. Valsakumar \\ Materials Science Group, Indira Gandhi Center for Atomic Research, Kalpakkam 603 102, Tamil Nadu, India
}

Axel van de Walle

Engineering and Applied Sciences Division, California Institute of Technology, Pasadena, California 91125, USA

(Received 27 November 2009; revised manuscript received 27 January 2010; published 18 March 2010)

\begin{abstract}
Phase stability of stable and metastable vanadium nitrides is studied using density functional theory (DFT) based total-energy calculations combined with cluster expansion Monte Carlo simulation and supercell methods. We have computed the formation enthalpy of the various stable and metastable vanadium nitride phases considering the available structural models and found that the formation enthalpies of the different phases decrease in the same order as they appear in the experimental aging sequence. DFT calculations are known to show stoichiometric $\mathrm{V}_{2} \mathrm{~N}$ to be polymorphic in $\epsilon-\mathrm{Fe}_{2} \mathrm{~N}$ and $\zeta-\mathrm{Fe}_{2} \mathrm{~N}$ structures within a few $\mathrm{meV}$ and $\mathrm{VN}$ to be more stable in $\mathrm{WC}\left(\mathrm{B}_{h}\right)$ phase than in the experimentally observed $\mathrm{NaCl}(\mathrm{B} 1)$ structure. As these nitrides are known to be generally nonstoichiometric due to presence of nitrogen vacancies, we used cluster expansion and supercell methods for examining the effect of nitrogen vacancies on the phase stability. It is found that nitrogen vacancies, represented by $\square$, stabilize $\epsilon$ - $\mathrm{Fe}_{2} \mathrm{~N}$ phase of $\mathrm{V}_{2} \mathrm{~N}_{1-x} \square_{x}$ and $\mathrm{NaCl}(\mathrm{B} 1)$ phase of $\mathrm{VN}_{1-x} \square_{x}$ compared to $\zeta-\mathrm{Fe}_{2} \mathrm{~N}$ and $\mathrm{WC}\left(\mathrm{B}_{h}\right)$ phases respectively, rendering the computed phase stability scenario to be in agreement with experiments. Analysis of supercell calculated electronic density of states (DOS) of $\mathrm{VN}_{1-x} \square_{x}$ with varying $x$, shows that the nitrogen vacancies increase the DOS at Fermi level in WC phase, whereas they decrease the $\mathrm{DOS}$ in $\mathrm{NaCl}$ phase. And this serves as the mechanism of enhancement of the stability of the $\mathrm{NaCl}$ phase. Monte Carlo simulations were used for computing the finite temperature formation enthalpies of these phases as a function of nitrogen-vacancy concentration and found close agreement for $\mathrm{NaCl}(\mathrm{B} 1)$ phase of $\mathrm{VN}_{1-x} \square_{x}$ for which measured values are available.
\end{abstract}

DOI: 10.1103/PhysRevB.81.104111

PACS number(s): 61.50.Lt, 64.75.Ef, 71.20.Be

\section{INTRODUCTION}

Vanadium nitrides constitute key precipitates in the design of creep resistant, high-strength Fe-Cr-based steels. ${ }^{1-3}$ Vanadium nitrides are also interesting in view of their potential application in hard coatings, catalysis, and optoelectronics. $^{4-9}$ In the vanadium-nitrogen system, three nitride phases, $\beta \mathrm{V}_{2} \mathrm{~N}_{1-x}\left(\epsilon-\mathrm{Fe}_{2} \mathrm{~N}\right.$ type $), \delta \mathrm{VN}_{1-x}(\mathrm{NaCl}$ type), and $\delta^{\prime} \mathrm{VN}_{1-x}$ have been identified and their crystal structures, ranges of homogeneity and melting or decomposition temperatures are determined. ${ }^{10}$ The variation in the composition of these phases is known to be due to vacancies on the nitrogen sublattice. ${ }^{10}$ Properties of these nitrides are known to depend strongly on their chemistry and structural state. ${ }^{11,12}$

The ground-state properties and phase stability of some of these phases have been studied in a previous work ${ }^{13}$ via density functional theory (DFT) based electronic-structure totalenergy calculations. Based on the calculated formation enthalpies, it is shown that stoichiometric $\mathrm{V}_{2} \mathrm{~N}$ is polymorphic in $\epsilon-\mathrm{Fe}_{2} \mathrm{~N}, \zeta-\mathrm{Fe}_{2} \mathrm{~N}$, and $\mathrm{Fe}_{2} \mathrm{C}$ structures within a few meV. $\mathrm{VN}$ is found to have enhanced stability in $\mathrm{WC}\left(\mathrm{B}_{h}\right)$ phase (by $180 \mathrm{meV} /$ atom) compared to the experimental $\mathrm{NaCl}(\mathrm{B} 1)$ structure. Supercell calculations of $\epsilon-\mathrm{Fe}_{2} \mathrm{~N}$-type $\mathrm{V}_{2} \mathrm{~N}$ and $\mathrm{NaCl}$-type $\mathrm{VN}$ with vacancies on the nitrogen sublattice have shown that nitrogen vacancies lower the energy leading to enhanced stability. However, the corresponding effect with respect to $\zeta-\mathrm{Fe}_{2} \mathrm{~N}$ and $\mathrm{WC}\left(\mathrm{B}_{h}\right)$ structures was not studied in the previous work. To further understand the origin of enhanced stability of stoichiometric VN in WC phase compared to $\mathrm{NaCl}$ phase, band-structure calculations were performed for $\mathrm{TiN}$ and $\mathrm{CrN}$ in $\mathrm{NaCl}$ as well as WC structures.
Although all the three substances are known to form in the $\mathrm{NaCl}$ structure, our DFT energetics shows that stoichiometric $\mathrm{CrN}$ also has low energy in WC phase like VN while TiN is seen to stabilize in $\mathrm{NaCl}$ structure. These observations indicate that the influence of nitrogen vacancies on the phase stability of vanadium nitrides should be studied more systematically. Recently, Lazar et al. (Ref. 3) have made a DFT study of stability of ternary $\mathrm{V}-\mathrm{Cr}-\mathrm{N}$ compounds with respect to decomposition into $\mathrm{V}-\mathrm{N}$ and $\mathrm{Cr}-\mathrm{N}$ binary nitrides. However, this work did not consider the stability of $\mathrm{VN}$ and $\mathrm{CrN}$, and $\mathrm{V}_{2} \mathrm{~N}$ between competing crystal structures, such as $\mathrm{WC}\left(\mathrm{B}_{h}\right)$ structure for $\mathrm{VN}$ and $\mathrm{CrN}$ and $\zeta-\mathrm{Fe}_{2} \mathrm{~N}$ structure for $\mathrm{V}_{2} \mathrm{~N}$. Also, they have not included the $\delta^{\prime} \mathrm{VN}_{1-x}$ phase as well as the various metastable vanadium nitrides that are known to form in the system.

In Ref. 13, the $\delta^{\prime} \mathrm{VN}_{1-x}$ was not considered due to the ambiguity regarding the crystal-structure data. ${ }^{10}$ Onozuka $^{14}$ identified $\delta^{\prime} \mathrm{VN}_{1-x}$ after prolonged annealing of $\delta \mathrm{VN}_{1-x}(0.18 \leq x \leq 0.26)$ at $500{ }^{\circ} \mathrm{C}$. This phase is found to have an ordered arrangement of $\mathrm{N}$ atoms with doubling of the lattice parameter of the $\mathrm{NaCl}$-type structure. This phase is proposed to have a crystal structure based on the space group $P 4_{2} / n m c$ (No. 137) with a total cell occupancy in the eightfold multiple cell of $32 \mathrm{~V}$ atoms and $26 \mathrm{~N}$ atoms. The proposed positions of the atoms in the structure model are reproduced in Table I.

In Table I, $\alpha, \beta, \gamma, \delta, \eta$, and $\zeta$ are the occupation probabilities of nitrogen atoms on each site and obey the constraint that $\alpha+\beta+2 \gamma+4 \delta+4 \eta+4 \zeta=16(1-x)$. From the diffraction intensities, values for $\alpha=\zeta=1, \beta=\frac{3}{4}, \gamma=\delta=\frac{7}{8}$, and 
TABLE I. Positions of the atoms in the structure model of $\delta^{\prime} \mathrm{VN}_{1-x}$ based on the space group $P 4_{2} / n m c$ (No. 137) with origin choice 1 (Ref. 14).

\begin{tabular}{lc}
\hline \hline $2 \alpha \mathrm{N}$ in 2(a) & $4 \mathrm{~V}$ in 4(c) with $z=\frac{1}{4}$ \\
$2 \beta \mathrm{N}$ in 2(b) & $4 \mathrm{~V}$ in $4(\mathrm{~d})$ with $z=\frac{1}{4}$ \\
$4 \gamma \mathrm{N}$ in 4(d) with $z=0$ & $8 \mathrm{~V}$ in $8(\mathrm{e})$ \\
$8 \delta \mathrm{N}$ in $8(\mathrm{f})$ with $x=\frac{1}{4}$ & $8 \mathrm{~V}$ in $8(\mathrm{~g})$ with $x=\frac{1}{4}$ \\
$8 \eta \mathrm{N}$ in $8(\mathrm{~g})$ with $x=\frac{1}{4}$ & $z=0$ \\
$z=\frac{1}{4}$ & $8 \mathrm{~V}$ in $8(\mathrm{~g})$ with $x=\frac{1}{4}$ \\
$8 \zeta \mathrm{N}$ in $8(\mathrm{~g})$ with $x=\frac{1}{4}$ & $z=\frac{1}{2}$ \\
$z=\frac{3}{4}$ & \\
\hline \hline
\end{tabular}

$\eta=\frac{1}{2}$ were derived for a composition of $\mathrm{VN}_{0.81}(x=0.19)$.

A series of metastable phases is also reported to form between the $\mathrm{V}$ terminal solid solution $(\mathrm{V})$ and $\beta \mathrm{V}_{2} \mathrm{~N}_{1-x}$. Carlson et al. ${ }^{10}$ have proposed a metastable phase diagram for the vanadium end of the V-N system in their review. Although the metastable phases are considered to involve ordering of interstitial $\mathrm{N}$ atoms in one or another distortion of the bcc lattice of elemental vanadium, their crystal structures have not been studied thoroughly. Therefore, in this paper, we examine the energetics of available structural models of $\delta^{\prime} \mathrm{VN}_{1-x}$ and metastable phases and investigate the phase stability of $\mathrm{V}_{2} \mathrm{~N}_{1-x}$ between $\epsilon-\mathrm{Fe}_{2} \mathrm{~N}$ and $\zeta-\mathrm{Fe}_{2} \mathrm{~N}$ lattices, and that of $\mathrm{VN}_{1-x}$ between $\mathrm{NaCl}(\mathrm{B} 1)$ and $\mathrm{WC}\left(\mathrm{B}_{h}\right)$ lattices within the pseudobinary model using cluster expansion Monte Carlo (MC) methods combined with DFT total-energy calculations.

Diverse reports concerning the number, structure, and stoichiometry of metastable phases of vanadium nitrides exist in the literature and the variations can be attributed to differing thermal histories, aging times, and differences in the sample compositions employed in the measurements. A typical aging sequence below $550{ }^{\circ} \mathrm{C}$ of a supersaturated solid solution of nitrogen in vanadium, $(\mathrm{V})$, is reported as below ${ }^{10}$

$$
\begin{aligned}
(\mathrm{V}) \rightarrow & (\mathrm{V})+\mathrm{V}_{16} \mathrm{~N} \rightarrow(\mathrm{V})+\mathrm{V}_{9} \mathrm{~N}_{2}\left(\text { or } \mathrm{V}_{9} \mathrm{~N}\right) \rightarrow(\mathrm{V}) \\
& +\mathrm{V}_{8} \mathrm{~N}\left(\text { or } \mathrm{V}_{9} \mathrm{~N}\right) \rightarrow(\mathrm{V})+\beta \mathrm{V}_{2} \mathrm{~N}_{1-x} .
\end{aligned}
$$

The inference of metastability of these phases is based on the time dependence of their formation even though slow kinetics during cooling to low temperatures can lead to their persistence for prolonged periods of time. The metastable phase with highest $\mathrm{V}$ concentration in this aging sequence with $\mathrm{V}_{16} \mathrm{~N}$ stoichiometry is found to have an unit cell 16 times larger than that of the bcc vanadium. ${ }^{10,15}$ This supercell is identified as a face-centered orthorhombic cell with $a_{\mathrm{s}}$ $=1.2140 \mathrm{~nm} \cong 4 a_{\mathrm{V}}, \quad b_{\mathrm{s}}=1.2504 \mathrm{~nm} \cong 4 a_{\mathrm{V}}, \quad$ and $c_{\mathrm{s}}$ $=0.3035 \mathrm{~nm} \cong a_{\mathrm{V}}$. From the composition and symmetry, the superlattice cell is inferred to contain 16 subcells and consists of $32 \mathrm{~V}$ atoms and $2 \mathrm{~N}$ atoms for a net stoichiometry of $\mathrm{V}_{16} \mathrm{~N}$ with the $\mathrm{N}$ atoms occupying the $(000)$ and $(1 / 2,1 / 2,1 / 2)$ superlattice sites. A bcc supercell was constructed based on this crystal structure data, in which, the $\mathrm{N}$ and $\mathrm{V}$ atoms are placed, respectively, at 2(a) and 32(o) Wyckoff sites of the space group $I 4 / \mathrm{mmm}$ (139). The 2(a) site has coordinates
(000) and $(1 / 2,1 / 2,1 / 2)$ as stated above. The $32(0)$ site corresponds to $(1 / 8,0,0)$ and its transformations.

The second metastable phase is reported to have bcc structure with a V sublattice parameter of $a_{\mathrm{V}}=0.3046 \mathrm{~nm}$, which required a supercell with $a_{\mathrm{s}}=0.9139=3 a_{\mathrm{V}}$, when combined with the $\mathrm{N}$ sublattice. This phase has I432 space group symmetry. Within symmetry constraints, there are three possibilities for the location of $\mathrm{N}$ atoms. ${ }^{16}$ One possibility utilizes tetrahedral sites and results in a stoichiometry of $\mathrm{V}_{9} \mathrm{~N}_{2}$, designated here as $\mathrm{V}_{9} \mathrm{~N}_{2}$ (Tet). The other two possibilities utilize octahedral sites but result in appreciably different stoichiometries, one with $\mathrm{V}_{9} \mathrm{~N}_{1}\left[\mathrm{~V}_{9} \mathrm{~N}_{1}(\right.$ Oct1) $)$ and the other with $\mathrm{V}_{9} \mathrm{~N}_{2}\left[\mathrm{~V}_{9} \mathrm{~N}_{2}(\right.$ Oct2) $)$. We have deduced the atomic coordinates of these three phases from the graphical description of the structure of these phases by Potter et al. (Ref. 16). Due to weak electron-diffraction intensities, it was very difficult to experimentally identify the correct structure from the three possibilities.

For the third metastable phase in the foregoing aging sequence, a variety of (incomplete) structures and stoichiometries have been proposed. We performed band-structure calculation using crystal-structure data proposed by Galkin et al. (Ref. 17). Based on neutron diffraction, they have reported an ordered phase with $a_{\mathrm{s}}=b_{\mathrm{s}}=2 \sqrt{2} a_{\mathrm{V}}$ and with $c_{\mathrm{s}}$ $=c_{\mathrm{V}}$. This phase has the space group $P 4_{2} / m n m$ with eight $\mathrm{V}$ in 8(i) with $x=0.625$ and $y=0.125$; four $\mathrm{V}$ in $4(\mathrm{f})$ with $x$ $=0.165$; four $\mathrm{V}$ in 4 (f) with $x=0.379$; and two $\mathrm{N}$ in 2 (a) for a net stoichiometry of $\mathrm{V}_{8} \mathrm{~N}$.

With regard to phase stability of $\beta \mathrm{V}_{2} \mathrm{~N}_{1-x} \square_{x}$ and $\delta \mathrm{VN}_{1-x} \square_{x}$, it is particularly important to examine the effect of nitrogen vacancies on the formation enthalpy between the two structures considered for each of the phases. Thus, the energetics of $\mathrm{V}_{2} \mathrm{~N}_{1-x} \square_{x}$ between $\epsilon$ - $\mathrm{Fe}_{2} \mathrm{~N}$ and $\zeta-\mathrm{Fe}_{2} \mathrm{~N}$ lattices and of $\mathrm{VN}_{1-x} \square_{x}$ between $\mathrm{NaCl}(\mathrm{B} 1)$ and $\mathrm{WC}\left(\mathrm{B}_{h}\right)$ lattices have to be computed as a function of nitrogen-vacancy concentration. We consider these phases as pseudobinary systems with nitrogen and structural vacancies constituting the two species keeping the occupancy of the $\mathrm{V}$ sublattice unaltered. Energetics of these phases is then examined within a first-principles framework employing a sublattice clusterexpansion (SCE) formalism which has been successfully used to model anion and/or cation disorder in ceramic systems as well as phase stability in pseudobinary transitionmetal alloys. ${ }^{18-20}$ The SCE involves energy calculations for several ordered superstructures representing various configurations of $\mathrm{N}$ atom and $\square$ on the nitrogen sublattices, from which values for the effective cluster interaction (ECI) parameters in a cluster expansion for the energy are extracted; with such a cluster expansion, Monte Carlo simulations are carried out to compute energy of the alloy as a continuous function of composition, temperature, and short-range order. In our previous paper [Ravi (Ref. 13)], it is seen that stoichiometric $\mathrm{V}_{2} \mathrm{~N}$ is polymorphic in $\epsilon-\mathrm{Fe}_{2} \mathrm{~N}$ and $\zeta-\mathrm{Fe}_{2} \mathrm{~N}$ structures with negligibly small energy difference, whereas the stoichiometric VN is found to have significantly low energy in $\mathrm{WC}\left(\mathrm{B}_{h}\right)$ phase (180 meV/atom) than experimental $\mathrm{NaCl}(\mathrm{B} 1)$ structure. It is also known that $\delta \mathrm{VN}_{1-x} \square_{x}$ has a wider homogeneity region than $\beta \mathrm{V}_{2} \mathrm{~N}_{1-x} \square_{x}$ phase. Therefore, for $\delta \mathrm{VN}_{1-x} \square_{x}$, we use both supercell and cluster expansion methods for examining the phase stability with varying concentration of nitrogen vacancies. 
In the next section, we provide further details related to the application of the SCE method to the study of phase stability in $\mathrm{V}_{2} \mathrm{~N}_{1-x} \square_{x}$ and $\mathrm{VN}_{1-x} \square_{x}$. Section III A presents the results of the direct calculations of crystal structures and energetics of $\delta^{\prime} \mathrm{VN}_{1-x}$ and metastable vanadium nitrides as well as results of supercell calculations for $\mathrm{VN}_{1-x} \square_{x}$ between $\mathrm{NaCl}(\mathrm{B} 1)$ and $\mathrm{WC}\left(\mathrm{B}_{h}\right)$ phases. The results of the cluster expansion Monte Carlo simulations are presented in Sec. III B. Summary and conclusions are given in the final section.

\section{COMPUTATIONAL METHODOLOGY}

\section{A. Cluster expansion and Monte Carlo simulations}

The cluster-expansion method is extensively used for the calculation of configuration dependence of the energy of alloys. ${ }^{20-34}$ In the cluster-expansion method as applied to a binary system comprising of $A$ and $B$, one selects an underlying parent lattice and defines a configuration $\sigma$ by specifying the occupations of each of the $n$ lattice sites by an $A$ atom or a $B$ atom. For each configuration, one assigns a set of spin variables, $\hat{S}_{i}(i=1,2, \ldots, n)$ to each of the $n$ sites with $\hat{S}_{i}=$ -1 or +1 depending on the site $i$ being occupied by an $A$ or $B$ atom, respectively. The problem of finding the energy of the $2^{n}$ possible configurations $\sigma$ can be exactly mapped onto a generalized Ising Hamiltonian

$$
\begin{aligned}
E_{C E}(\sigma)= & J_{0}+\sum_{i} J_{i} \hat{S}_{i}(\sigma)+\sum_{j<i} J_{i j} \hat{S}_{i}(\sigma) \hat{S}_{j}(\sigma) \\
& +\sum_{k<j<i} J_{i j k} \hat{S}_{i}(\sigma) \hat{S}_{j}(\sigma) \hat{S}_{k}(\sigma)+\cdots,
\end{aligned}
$$

where the $J$ 's are the interaction energies (effective cluster interactions) of various orders and the second term is a summation over all sites in the lattice, the third over all pairs of sites, the fourth over all triplets, and so on. The interaction energies possess the symmetry properties of the parent lattice, and thus, all terms with equal coefficients in Eq. (1) may be grouped together. Consequently, Eq. (1) can be written more compactly as

$$
E_{C E}(\sigma)=\sum_{f} D_{f} J_{f} \bar{\Pi}_{f}(\sigma),
$$

where $f$ refers to symmetry-distinct cluster of atoms defined as a figure comprising of several lattice sites (e.g., pairs, triplets, etc.). $D_{f}$ is the number of figures per lattice site, $J_{f}$ is the Ising-type interaction energy for the figure $f$, and the "lattice-averaged product" $\bar{\Pi}_{f}$ is defined as a product of the variables $\hat{S}_{i}$, for all sites of the figure $f$ and the overbar denotes an average over all symmetry equivalent figures

$$
\bar{\prod}_{f}(\sigma)=\frac{1}{N_{L}} \sum_{\hat{R}}^{N_{L}} \prod_{\hat{R} f}(\sigma),
$$

where the summation is over the $N_{L}$ space group operations $(\hat{R})$ of the lattice. The completeness and orthonormality of the set $\left\{\Pi_{f}(\sigma)\right\}$ of spin products in the space of all configurations assure that expansions in Eqs. (1) and (2) are exact if

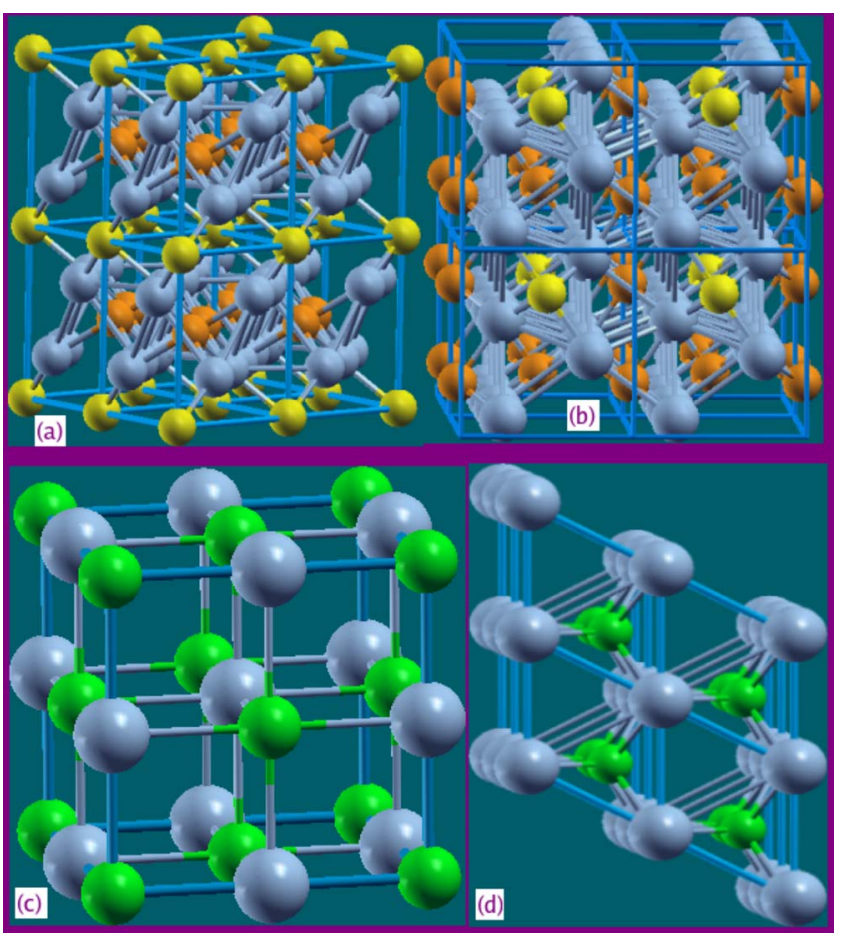

FIG. 1. (Color online) Sketch of unit cells of structures considered for $\mathrm{V}_{2} \mathrm{~N}$ and $\mathrm{VN}$. Here (a) is $\epsilon-\mathrm{Fe}_{2} \mathrm{~N}$ structure and (b) is $\zeta-\mathrm{Fe}_{2} \mathrm{~N}$ structure. In the bottom row, (c) and (d) are $\mathrm{NaCl}(\mathrm{B} 1)$ and $\mathrm{WC}\left(\mathrm{B}_{h}\right)$ structures of VN. For illustration of sublattice cluster expansion, atoms on the $\mathrm{N}$ sublattice are indicated by yellow and orange balls, respectively. In (a), the yellow atoms on the nitrogen sublattice are active sites for $\mathrm{N} / \square$ substitution while orange atoms are frozen. In the other structures, entire nitrogen sublattice is active for $\mathrm{N} / \square$ substitution.

all $2^{n}$ figures are used to describe all the $2^{n}$ configurational energies.

While mathematical completeness requires that all $2^{n}$ terms be included in Eqs. (1) and (2), physical intuition suggests that some interactions are less important than the others, and thus can be neglected. It is known that generally good accuracy can be obtained by retaining only $n_{F} \ll 2^{n}$ interactions. ${ }^{25,26,30}$ Then, following Connoly and Williams, ${ }^{21}$ total energies of $n_{\sigma}$ structures are calculated directly $\left[E_{\text {direct }}(\sigma)\right.$ or $\left.E_{Q M}(\sigma)\right]$ and the set of interactions $\left\{J_{f}\right\}$ are obtained by singular value decomposition (SVD) of the following expression:

$$
\sum_{\sigma}^{n_{\sigma}}\left|\sum_{f}^{n_{F}} D_{f} J_{f}^{C W} \bar{\Pi}_{f}(\sigma)-E_{\text {direct }}(\sigma)\right|^{2}=\text { minimum. }
$$

This leads to

$$
J_{f}^{C W}=\frac{1}{D_{f}} \sum_{\sigma}^{n_{\sigma}}\left[\tilde{\bar{\Pi}}^{-1}\right]_{\sigma, f} E_{\text {direct }}(\sigma) .
$$

In Eq. (5), $\widetilde{\bar{\Pi}}$ represents the (in general rectangular) $n_{\sigma} \times n_{F}$ matrix with elements $\bar{\Pi}_{f}(\sigma)$ and $\left[\tilde{\bar{\Pi}}^{-1}\right]_{\sigma, f}$ is the $(\sigma, f)$ element 
TABLE II. First-principles calculated (US-GGA) structural and thermodynamic properties of stable and metastable vanadium nitrides. $T=0 \mathrm{~K}$ formation enthalpies, $\Delta H$, are given in meV/atom.

\begin{tabular}{|c|c|c|c|}
\hline \multirow[b]{2}{*}{ Phase } & \multicolumn{2}{|c|}{$\begin{array}{l}\text { Lattice parameters }(\mathrm{a}, \mathrm{b}, \mathrm{c}) \\
(\AA)\end{array}$} & \multirow{2}{*}{$\begin{array}{c}\begin{array}{c}\Delta H \\
\text { (meV/atom) }\end{array} \\
\text { US-GGA }\end{array}$} \\
\hline & US-GGA & Expt. (Ref. 10) & \\
\hline $\mathrm{V}_{32} \mathrm{~N}_{26}\left(\delta^{\prime} \mathrm{VN}_{1-x}\right)$ & $8.14,8.22$ & 8.28 & -1028.7 \\
\hline $\mathrm{V}_{32} \mathrm{~N}_{2}\left(\mathrm{~V}_{16} \mathrm{~N}_{1}\right)$ & $12.16,12.16,2.98$ & $12.14,12.50,3.04$ & -155.8 \\
\hline $\mathrm{V}_{54} \mathrm{~N}_{6}-\mathrm{V}_{9} \mathrm{~N}_{1}$ (Oct1) & 9.12 & 9.14 & -267.7 \\
\hline $\mathrm{V}_{54} \mathrm{~N}_{12}-\mathrm{V}_{9} \mathrm{~N}_{2}$ (Oct2) & 9.41 & 9.14 & -130.5 \\
\hline $\mathrm{V}_{54} \mathrm{~N}_{12}-\mathrm{V}_{9} \mathrm{~N}_{2}$ (Tet) & 9.38 & 9.14 & -260.3 \\
\hline $\mathrm{V}_{8} \mathrm{~N}\left(\mathrm{~V}_{16} \mathrm{~N}_{2}\right)$ & $8.71,2.97$ & $8.58,3.04$ & -335.0 \\
\hline
\end{tabular}

of its inverse in case $n_{\sigma}=n_{F}$ and the pseudoinverse (obtained via SVD) if $n_{\sigma}>n_{F}$.

This means, if a hierarchy of interactions exists such that $n_{F} \ll 2^{n}$ interactions can describe $E_{C E}(\sigma)[$ Eq. (2)] with reasonable precision [underlying the calculation of $E_{Q M}(\sigma)$ ], then the energy of any of the $2^{n}$ configurations can be calculated almost immediately by calculating the spin products and summing Eq. (2). Thus, after an initial investment of calculating $n_{\sigma}$ total energies $\left\{E_{Q M}(\sigma)\right\}$ using $a b$ initio method, one could predict the energy $E\left(\sigma^{\prime}\right)$ of any of the remaining $2^{n}-n_{\sigma} \simeq 2^{n}$ configurations

$$
E_{C E}\left(\sigma^{\prime}\right)=\sum_{\sigma}^{n_{\sigma}} A\left(\sigma, \sigma^{\prime}\right) E_{\text {direct }}(\sigma) \text {, }
$$

where

$$
A\left(\sigma, \sigma^{\prime}\right)=\sum_{f}^{n_{F}} \bar{\Pi}_{f}\left(\sigma^{\prime}\right)\left[\tilde{\bar{\Pi}}^{-1}\right]_{\sigma, f^{\prime}}
$$

Equation (6) then establishes the existence of approximate (i.e., within a tolerance, $\delta E$ ) linear dependencies among the

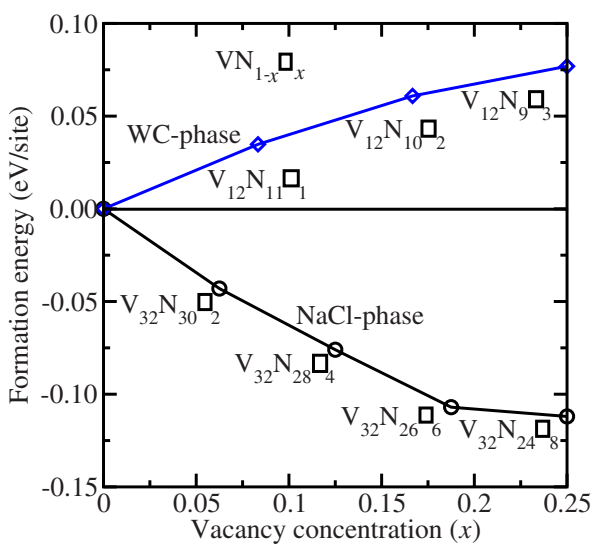

FIG. 2. (Color online) Formation enthalpy of pseudobinary $\mathrm{VN}_{1-x} \square_{x}$ with respect to $\mathrm{VN}$ and $\mathrm{V} \square$ for $\mathrm{NaCl}$ and $\mathrm{WC}$ structures obtained from supercell calculations. Nitrogen vacancies lower the formation enthalpy of $\mathrm{NaCl}$ phase while they increase the formation enthalpy of WC phase although this phase has low formation enthalpy at stoichiometric composition (see Fig. 3). energies of different configurations. A converged cluster expansion within a prescribed tolerance $\delta E$ can then be used to obtain the energy of any of the $2^{n}$ possible configurations, at virtually no additional computational cost.

A SCE takes the same form as Eq. (2) and formally, each ECI is also exactly given by Eqs. (4) and (5). As stated previously, in this work, we construct cluster expansion for $\mathrm{V}_{2} \mathrm{~N}_{1-x} \square_{x}$ between $\epsilon-\mathrm{Fe}_{2} \mathrm{~N}$ and $\zeta-\mathrm{Fe}_{2} \mathrm{~N}$ lattices and for $\mathrm{VN}_{1-x} \square_{x}$ between $\mathrm{NaCl}(\mathrm{B} 1)$ and $\mathrm{WC}\left(\mathrm{B}_{h}\right)$ lattices, allowing "substitution" of $\square$ in the $\mathrm{N}$ sublattice(s) while in the other sublattice $\mathrm{V}$ acts as a spectator species. The spectator atoms do not explicitly enter the expression of the cluster expansion because their configuration on their respective sublattice

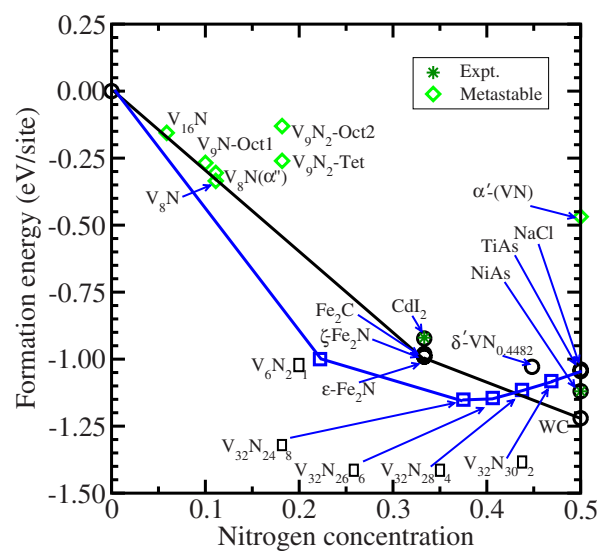

FIG. 3. (Color online) Formation enthalpy of stable and metastable vanadium nitrides as a function of concentration. Data represented by circles and diamonds are with respect to bcc vanadium and isolated $\mathrm{N}_{2}$ molecule. Data represented by square are obtained by combining the vacancy-induced decrease in formation energy (from Fig. 2) with the formation energy of NaCl-type stoichiometric $\mathrm{VN}$. The data point with $\mathrm{V}_{6} \mathrm{~N}_{2} \square_{1}$ label corresponds to $\epsilon$-Fe ${ }_{2} \mathrm{~N}$ type $\mathrm{V}_{2} \mathrm{~N}$ phase, obtained using a similar procedure. Tie lines are drawn connecting low-energy structures, one including structures with nitrogen vacancies and another neglecting structures with nitrogen vacancies. Experimental values are from Ref. 10. This figure is actually an updated version presented in a previous work [Ravi (Ref. 13)] and the reader may refer to this paper for details on the phases for which more information is required. It may be noted that calculated equilibrium formation enthalpies decrease in the same order as the aging sequence presented in Sec. I. 
Fitted energies for $\mathrm{V}_{6} \mathrm{~N}_{3-x} \mathrm{a}_{x}\left(\varepsilon-\mathrm{Fe}_{2} \mathrm{~N}\right.$-type $)$

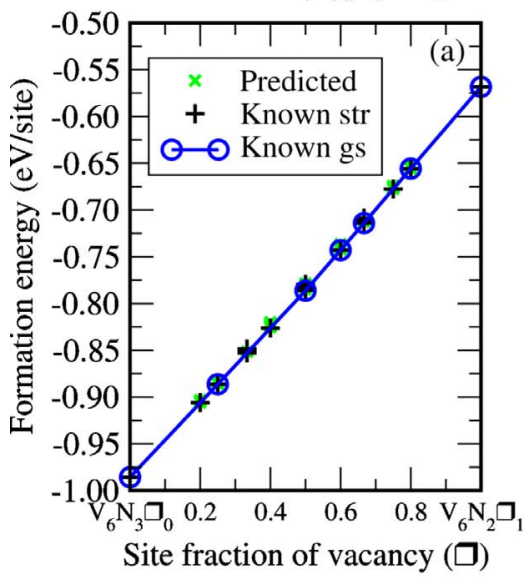

Fitted energies for $\mathrm{VN}_{1-x} \square_{x}(\mathrm{NaCl}$-type $)$

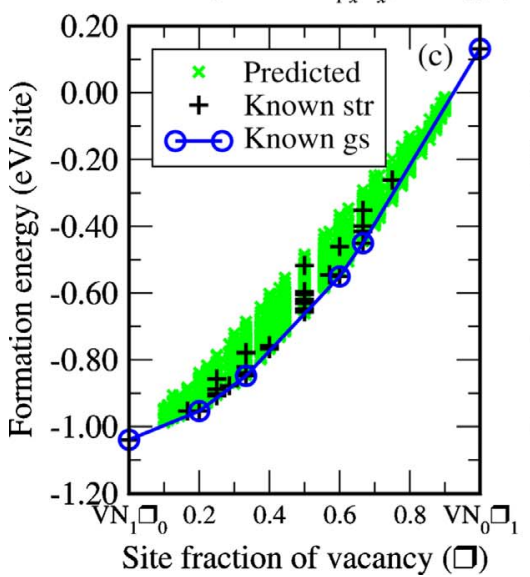

Fitted energies for $\mathrm{V}_{8} \mathrm{~N}_{4-x} \square_{x}\left(\zeta-\mathrm{Fe}_{2} \mathrm{~N}\right.$-type $)$

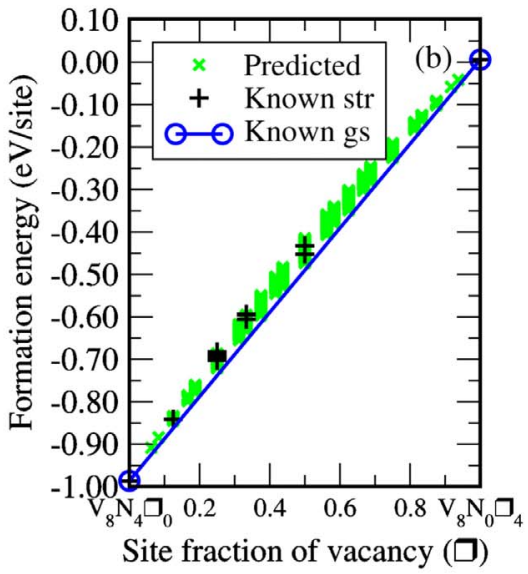

Fitted energies for $\mathrm{VN}_{1-x} \square_{x}$ (WC-type)

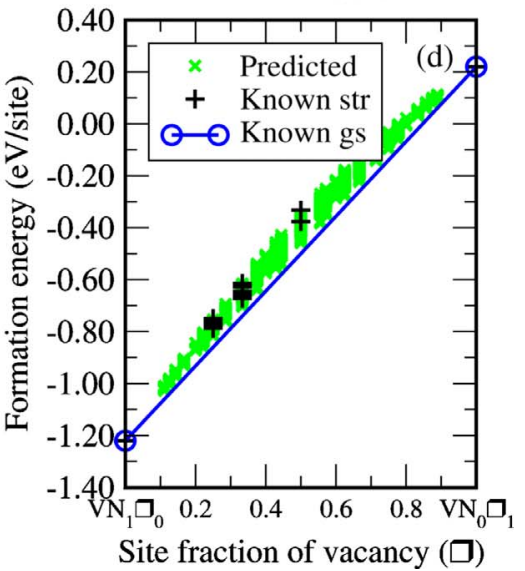

FIG. 4. (Color online) Cluster expansion and direct enumeration ground-state search results of formation energies of (a) $\epsilon$-Fe ${ }_{2} \mathrm{~N}$-type $\mathrm{V}_{2} \mathrm{~N}_{1-x} \square_{x}$, (b) $\zeta-\mathrm{Fe}_{2} \mathrm{~N}$-type $\mathrm{V}_{2} \mathrm{~N}_{1-x} \square_{x}$, (c) $\mathrm{NaCl}\left(\mathrm{B} 1\right.$ )-type $\mathrm{VN}_{1-x} \square_{x}$, and (d) $\mathrm{WC}\left(\mathrm{B}_{h}\right.$ )-type $\mathrm{VN}_{1-x} \square_{x}$ pseudobinary systems. Circles denote the energies predicted from the cluster expansion for which the ab initio energy is known. The dotted line indicates the tie line connecting low-energy configurations from which the ground states can be identified. The crosses denote predicted energies for a large database of generated structures used to verify the ground states. Cluster expansion based on $\epsilon-\mathrm{Fe}_{2} \mathrm{~N}$ lattice covered all structures with at most 45 atoms per unit cell. The $\zeta-\mathrm{Fe}_{2} \mathrm{~N}$ cluster expansion covered structures with 48 atoms per unit cell. Cluster expansion based on $\mathrm{NaCl}$ and WC lattices covered structures with 20 and 18 atoms per unit cell, respectively. The $\epsilon$-Fe $\mathrm{N}_{2}$-type $\mathrm{V}_{2} \mathrm{~N}_{1-x} \square_{x}$ cluster expansion used $\mathrm{V}_{6} \mathrm{~N}_{3} \square_{0}$ and $\mathrm{V}_{6} \mathrm{~N}_{2} \square$. as reference states for the calculation of formation energies. $\mathrm{V}_{8} \mathrm{~N}_{4} \square_{0}$ and $\mathrm{V}_{8} \mathrm{~N}_{0} \square_{4}$ are used as reference states for $\zeta$-Fe $\mathrm{Fe}_{2} \mathrm{~N}$-type $\mathrm{V}_{2} \mathrm{~N}_{1-x} \square$. For $\mathrm{NaCl}(\mathrm{B} 1)$ and $\mathrm{WC}\left(\mathrm{B}_{h}\right)$-type $\mathrm{VN}_{1-x} \square_{x}$, we used $\mathrm{VN}_{1} \square_{0}$ and $\mathrm{VN}_{0} \square_{1}$ as reference states. Energies of reference states are made of sum of energies of corresponding number of bcc $\mathrm{V}$ and of $\mathrm{N}$ of $\mathrm{N}_{2}$ molecule so that the formation energies in the plots are with respect to bcc $\mathrm{V}$ and isolated $\mathrm{N}_{2}$ molecule.

does not change. However, their presence modifies the construction of the cluster expansion in two important ways. First, the energy $E(\sigma)$ in Eqs. (2), (4), and (5) includes the contribution of the spectator species so that the ECI coupling two sites also includes the indirect interaction between these sites mediated through the spectator specie. The second effect of the spectator species is that they lower the symmetry of the sublattice of interest so that the ECI associated with two clusters that have apparently identical geometries may still differ due to differing environments. Formally, cluster equivalence is thus determined according to the joint space group of the two sublattices. ${ }^{20}$ The conventional unit cells considered for $\mathrm{V}_{2} \mathrm{~N}_{1-x} \square_{x}$ and $\mathrm{VN}_{1-x} \square_{x}$ are illustrated in Fig. 1 , where atoms on the $\mathrm{N}$ sublattice are indicated by yellow and orange balls, respectively.
In Fig. 1(a), the positions of the yellow atoms on the nitrogen sublattice are active sites for $\mathrm{N} / \square$ substitution while orange atoms are frozen. In the other structures, entire nitrogen sublattice is active for $\mathrm{N} / \square$ substitution. The $\epsilon-\mathrm{Fe}_{2} \mathrm{~N}$ structure has three formula units in the unit cell resulting in the cell stoichiometry of $\mathrm{V}_{6} \mathrm{~N}_{3}$, and is based on the $P \overline{3} 1 m$ space group symmetry with six $\mathrm{V}$ atoms in $6(\mathrm{k})$, two (orange) $\mathrm{N}$ in 2(d), and one (yellow) in 1(a) positions. Cluster expansion was constructed with reference to $\mathrm{V}_{6} \mathrm{~N}_{3}$ and $\mathrm{V}_{6} \mathrm{~N}_{2} \square_{1}$, allowing $\mathrm{N} / \square$ substitution on the 1 (a) sites and corresponds to sublattice cluster expansion of $\mathrm{V}_{2} \mathrm{~N}_{1-x} \square_{x}$ for $x=0$ to $1 / 3$. Concentration range used for ground-state checking was $[0,1 / 2]$. The $\zeta-\mathrm{Fe}_{2} \mathrm{~N}$-type phase is based on the $\mathrm{Pbcn}$ space group and has four formula units in the unit cell with $\mathrm{V}$ atoms at $8(\mathrm{~d})$ and $\mathrm{N}$ atoms at $4(\mathrm{c})$ sites. The cluster expan- 
TABLE III. ECI's parameters of $\mathrm{V}_{2} \mathrm{~N}_{1-x} \square_{x}$. The empty, point, and pair clusters are labeled as $J(0,1)$, $J(1,1)$, and $J(2,1, \ldots, n)$, respectively. Coordinates are in fraction of lattice vectors. Cluster size is defined as the length of the longest pair contained in the cluster. In the cluster expansion of $\epsilon-\mathrm{Fe}_{2} \mathrm{~N}$ phase, 22 input structures were used with a maximum of 45 atoms in the unit cell and has given a cross validation $\mathrm{CV}$ $=6 \mathrm{meV}$. Cluster expansion of $\zeta-\mathrm{Fe}_{2} \mathrm{~N}$ phase used 25 input structures with a maximum of 48 atoms in the unit cell and has given a $\mathrm{CV}=31 \mathrm{meV}$.

\begin{tabular}{|c|c|c|c|c|}
\hline Cluster & Coordinates & $\begin{array}{c}\text { Size } \\
(\AA)\end{array}$ & Multiplicity & ECI (eV/cluster) \\
\hline \multicolumn{5}{|c|}{$\epsilon$ - $\mathrm{Fe}_{2} \mathrm{~N}$-type lattice } \\
\hline$J(0,1)$ & & & & -7.033 \\
\hline$J(1,1)$ & $(1.0,1.0,1.0)$ & & 1 & 1.876 \\
\hline$J(2,1)$ & $(1.0,1.0,1.0)(1.0,1.0,2.0)$ & 4.560 & 1 & 0.021 \\
\hline$J(2,2)$ & $(1.0,1.0,1.0)(1.0,0.0,1.0)$ & 4.910 & 3 & -0.001 \\
\hline$J(2,3)$ & $(1.0,1.0,1.0)(1.0,0.0,0.0)$ & 6.701 & 3 & 0.004 \\
\hline$J(2,4)$ & $(1.0,1.0,1.0)(2.0,1.0,0.0)$ & 6.701 & 3 & 0.003 \\
\hline \multicolumn{5}{|c|}{$\zeta$-Fe ${ }_{2} \mathrm{~N}$-type lattice } \\
\hline$J(0,1)$ & & & & -5.256 \\
\hline$J(1,1)$ & $(1.0,0.3732,0.25)$ & & 4 & 1.486 \\
\hline$J(2,1)$ & $(1.0,0.6268,0.75)(1.0,0.3732,1.25)$ & 2.848 & 4 & -0.012 \\
\hline$J(2,2)$ & $(1.0,0.6268,0.75)(0.5,0.8732,1.25)$ & 3.624 & 8 & -0.029 \\
\hline$J(2,3)$ & $(0.5,0.1268,0.75)(1.0,-0.3732,0.75)$ & 3.648 & 8 & -0.025 \\
\hline$J(2,4)$ & $(1.0,0.6268,0.75)(0.0,0.6268,0.75)$ & 4.533 & 4 & -0.001 \\
\hline$J(2,5)$ & $(1.0,0.6268,0.75)(1.0,0.6268,-0.25)$ & 4.904 & 4 & -0.004 \\
\hline$J(2,6)$ & $(1.0,0.3732,0.25)(1.0,-0.3732,-0.25)$ & 4.922 & 4 & -0.022 \\
\hline
\end{tabular}

sion was constructed with reference to $\mathrm{V}_{8} \mathrm{~N}_{4} \square_{0}$ and $\mathrm{V}_{8} \mathrm{~N}_{0} \square_{4}$, allowing $\mathrm{N} / \square$ substitution on the sites corresponding to 4(c) positions and corresponds to sublattice cluster expansion of $\mathrm{V}_{2} \mathrm{~N}_{1-x} \square_{x}$ for $x=0$ to 1 . Concentration range used for ground-state checking was $[0,1 / 3]$. And for $\mathrm{NaCl}(\mathrm{B} 1)$ - and $\mathrm{WC}\left(\mathrm{B}_{h}\right)-\mathrm{VN}_{1-x} \square_{x}$, cluster expansions were constructed with reference to $\mathrm{VN}$ and $\mathrm{V} \square$. Concentration range used for ground-state checking were $[0,1 / 2]$ and $[0,1 / 3]$, respectively.

The cluster expansions were constructed using the alloy theoretic automated toolkit (ATAT). ATAT proceeds by gradually increasing the number of clusters included in the cluster expansion until the desired accuracy is achieved. For a given set of structural energies, the optimal set of clusters is determined by minimizing the cross-validation (CV) score, defined as $\left[\Sigma_{s}\left(E_{s}-\hat{E}_{\bar{S}}\right)^{2}\right]^{(1 / 2)}$, where $E_{s}$ is the energy of structure $s$ while $\hat{E}_{\bar{s}}$ is the energy predicted from the cluster expansion using all structures except the structure $s$. The database of structures used in the fit of the cluster expansion is gradually enlarged by adding structures different from the structures already considered in terms of the correlations $\sigma_{\alpha}$.

In all the present cluster expansions, the energy $\Delta E(\sigma)$ of a given configuration $(\sigma)$ will be given as an excess energy, which may be identified as the energy of formation of the specific configuration

$$
\Delta E(\sigma)=E(\sigma)-[(1-x) E(A)+x E(B)],
$$

where, for example, in the case of $\epsilon-\mathrm{Fe}_{2} \mathrm{~N}$ type $\mathrm{V}_{2} \mathrm{~N}_{1-x} \square_{x}, A$ is $\mathrm{V}_{6} \mathrm{~N}_{3}, B$ is $\mathrm{V}_{6} \mathrm{~N}_{2} \square_{1}$, and $x$ is the number of $\mathrm{V}_{6} \mathrm{~N}_{2} \square_{1}$ in the system $(\sigma)$. Energies of these reference states are made up of sum of energies of corresponding number of bcc $\mathrm{V}$ and of $\mathrm{N}_{2} / 2$. As the cluster expansion proceeds, the formation energies of the structures used for the cluster expansion are plotted as function of concentration and configurations of the system being studied. The ground states of the system are identified by the points on the convex hull of formation energy. The ground-state search was carried out by exhaustive enumeration by a direct determination of all possible configurations. ${ }^{35}$ Formally such an approach is impossible for very large unit cells as there are prohibitively large number of states to enumerate. Direct enumeration method is both possible and very useful when applied to relatively small unit cell consisting of about 50 atoms and we have applied this method in this work.

Having constructed the cluster-expansion Hamiltonian, it is used to perform Monte Carlo simulations in order to obtain the heats of formation as a function of composition and temperature. The Monte Carlo simulations were performed using the program EMC2 of ATAT described elsewhere. ${ }^{36}$ EMC2 samples a semigrand canonical ensemble in which chemical potential $\Delta \mu$, temperature $T$, and pressure $P$ are specified with a conserved total number $M\left(=\sum_{i=1}^{c} M_{i}\right)$ of particles. The composition or the molar fraction $x \equiv\left\{\frac{M_{i}}{M} ; i\right.$ $=2, \ldots, c\}$ is allowed to vary with a constraint of $\sum_{i=1}^{c} M_{i}$ $=M$ being fixed. The thermodynamic integration method was used to determine the grand canonical potential $\phi^{\alpha}$ of each phase $\alpha$ as a function of chemical potential $\Delta \mu$. The grand canonical potential at the starting point of the integration path was obtained from the high-temperature series expan- 
TABLE IV. ECI's parameters of $\mathrm{VN}_{1-x} \square_{x}$ in $\mathrm{NaCl}$ (B1)-type lattice. The empty, point, pair, and threebody clusters are labeled as $J(0,1), J(1,1), J(2,1 \ldots, n)$, and $J(3,1 \ldots, n)$, respectively. Coordinates are in fraction of lattice vectors. Cluster size is defined as the length of the longest pair contained in the cluster. This calculation used 35 structures (with a maximum of 20 atoms in the unit cell) as input in the cluster expansion fit and has given a $\mathrm{CV}=77 \mathrm{meV}$.

\begin{tabular}{lcccc}
\hline \hline Cluster & Coordinates & $\begin{array}{c}\text { Size } \\
(\AA)\end{array}$ & Multiplicity & ECI (meV/cluster) \\
\hline$J(0,1)$ & $(1.0,1.0,1.0)$ & & -1.224 \\
$J(1,1)$ & $(1.0,1.0,1.0)(1.5,1.0,0.5)$ & 2.920 & 6 & 1.098 \\
$J(2,1)$ & $(1.0,1.0,1.0)(0.0,1.0,1.0)$ & 4.130 & 3 & 0.040 \\
$J(2,2)$ & $(1.0,1.0,1.0)(0.5,2.0,0.5)$ & 5.058 & 12 & 0.013 \\
$J(2,3)$ & $(1.0,1.0,1.0)(0.0,1.0,2.0)$ & 5.840 & 0.000 \\
$J(2,4)$ & $(1.0,1.0,1.0)(2.5,1.0,0.5)$ & 6.530 & 12 & 0.014 \\
$J(2,5)$ & $(1.0,1.0,1.0)(1.5,1.0,0.5)(1.5,0.5,1.0)$ & 2.920 & 8 & -0.004 \\
$J(3,1)$ & $(1.0,1.0,1.0)(0.5,0.5,1.0)(0.0,1.0,1.0)$ & 4.130 & 12 & -0.012 \\
$J(3,2)$ & $(1.0,1.0,1.0)(0.5,1.0,0.5)(0.5,2.0,0.5)$ & 5.058 & 24 & 0.006 \\
$J(3,3)$ & $(1.0,1.0,1.0)(1.5,1.5,1.0)(0.5,2.0,0.5)$ & 5.058 & 24 & -0.002 \\
$J(3,4)$ & $(1.0,1.0,1.0)(0.5,1.5,1.0)(0.5,2.0,0.5)$ & 5.058 & 24 & 0.002 \\
$J(3,5)$ & $(1.0,1.0,1.0)(0.0,1.0,1.0)(0.5,2.0,0.5)$ & 5.058 & 24 & 0.000 \\
$J(3,6)$ & $(1.0,1.0,1.0)(1.5,1.5,0.0)(0.5,2.0,0.5)$ & 5.058 & 8 & 0.007 \\
$J(3,7)$ & $(1.0,1.0,1.0)(0.5,0.5,1.0)(0.0,1.0,2.0)$ & 5.840 & 48 & 0.002 \\
$J(3,8)$ & $(1.0,1.0,1.0)(0.5,1.0,1.5)(0.0,1.0,2.0)$ & 5.840 & 6 & -0.003 \\
$J(3,9)$ & $(1.0,1.0,1.0)(0.0,1.0,1.0)(0.0,1.0,2.0)$ & 5.840 & 12 & -0.006 \\
$J(3,10)$ & $(1.0,1.0,1.0)(0.5,0.0,1.5)(0.0,1.0,2.0)$ & 5.840 & 12 & -0.001 \\
$J(3,11)$ & $(1.0,1.0,1.0)(0.0,2.0,1.0)(0.0,1.0,2.0)$ & 5.840 & 8 & 0.003 \\
$J(3,12)$ & & & 0.010 \\
\hline \hline
\end{tabular}

sion or low-temperature series expansion. For some of the phases studied in this work, our Monte Carlo simulations could not find stable equilibrium configurations for practical temperatures. In such cases, it is natural to assume that shortrange order to be negligible and compute their formation enthalpy in the limit of complete disorder using BraggWilliams approximation given by

$$
\Delta E(x)=\sum_{f} D_{f} J_{f}(2 x-1)^{n_{f}},
$$

where $n_{f}$ is the number of sites in $f$. Here we have used the fact that the average cluster functions $\left\langle\sigma_{f}\right\rangle=\Pi_{i \in f}\left\langle\sigma_{i}\right\rangle=(2 x$ $-1)^{n_{f}}$ when the occupation of the sites is completely random.

\section{B. Density-functional-theory calculations}

The electronic-structure total-energy calculations of $\delta^{\prime} \mathrm{VN}_{1-x}$, metastable phases, supercells of $\mathrm{NaCl}$ and $\mathrm{WC}$ type $\mathrm{VN}_{1-x} \square_{x}$ as well as input structures required for cluster expansion were performed using pseudopotential method and a plane-wave-basis set, as implemented in the VASP code. ${ }^{37,38}$ VASP solves the Kohn-Sham equations self-consistently. For the present study, the one-electron wave functions were expanded in a plane-wave basis with a $400 \mathrm{eV}$ energy cutoff and the interaction of valence electrons with the core electrons described by ultrasoft (US) pseudopotentials. ${ }^{39}$ The generalized gradient approximation (GGA) of Perdew ${ }^{40}$ was used for the exchange-correlation energy functional. We use the first-order Methfessel-Paxton method ${ }^{41}$ of electronic occupancy with a smearing width of $0.1 \mathrm{eV}$. Brillouin-zone integrations use Monkhorst-Pack $k$-points mesh. ${ }^{42}$ The choices for plane-wave energy cutoff, exchange-correlation functional and $k$-points mesh are described in our previous work. $^{13}$ For the cluster-expansion calculations, we set KPPRA $=8000$. KPPRA automatically set the $k$-point mesh for similar systems. ${ }^{43}$ For NaCl-type VN, KPPRA $=8000$ translates to $16 \times 16 \times 16 k$-points grid.

\section{RESULTS AND DISCUSSION}

\section{A. Energetics of stable and metastable vanadium nitrides}

First, the directly calculated energetics of the stable and metastable vanadium nitrides is presented. Table II gives the calculated equilibrium lattice parameters and formation enthalpy of the various phases considered in the direct DFT calculations. For the calculation of energies of $\delta^{\prime} \mathrm{VN}_{1-x}$ phase, based on the lowest nitrogen occupation probabilities (Table I), we chose to have four of $8(\mathrm{~g})$ (with $x=z=1 / 4$ ) and both of 2(b) sites vacant so that the superlattice cell corresponds to $\mathrm{V}_{32} \mathrm{~N}_{26}$. It is evident that the calculated lattice parameter is in good agreement with experimental value (within 1.3\%). No experimental value of formation enthalpy is available for comparison with our calculated value of $\Delta H=-1029 \mathrm{meV} /$ atom. This is of the same order as that of 
TABLE V. ECI's parameters of $\mathrm{VN}_{1-x} \square_{x}$ in $\mathrm{WC}\left(\mathrm{B}_{h}\right)$-type lattice. The empty, point, and pair clusters are labeled as $J(0,1), J(1,1)$, and $J(2,1 \ldots, n)$, respectively. Coordinates are in fraction of lattice vectors. Cluster size is defined as the length of the longest pair contained in the cluster. This calculation used 23 structures (with a maximum of 18 atoms in the unit cell) as input in the cluster expansion fit and has given a CV $=50 \mathrm{meV}$.

\begin{tabular}{lcccc}
\hline \hline Cluster & Coordinates & $\begin{array}{c}\text { Size } \\
(\AA)\end{array}$ & Multiplicity & ECI (meV/cluster) \\
\hline$J(0,1)$ & $(2 / 3,1 / 3,1 / 2)$ & & 1 & -0.794 \\
$J(1,1)$ & $(2 / 3,1 / 3,1 / 2)(2 / 3,1 / 3,3 / 2)$ & 2.650 & 1 & 1.442 \\
$J(2,1)$ & $(2 / 3,1 / 3,1 / 2)(5 / 3,1 / 3,1 / 2)$ & 2.750 & 3 & 0.016 \\
$J(2,2)$ & $(2 / 3,1 / 3,1 / 2)(5 / 3,1 / 3,-1 / 2)$ & 3.820 & 6 & -0.014 \\
$J(2,3)$ & $(2 / 3,1 / 3,1 / 2)(-1 / 3,4 / 3,1 / 2)$ & 4.763 & 3 & -0.020 \\
$J(2,4)$ & $(2 / 3,1 / 3,1 / 2)(2 / 3,1 / 3,-3 / 2)$ & 5.300 & 1 & -0.004 \\
$J(2,5)$ & $(2 / 3,1 / 3,1 / 2)(-1 / 3,4 / 3,-1 / 2)$ & 5.450 & 6 & 0.004 \\
$J(2,6)$ & $(2 / 3,1 / 3,1 / 2)(2 / 3,-5 / 3,1 / 2)$ & 5.500 & 3 & -0.004 \\
$J(2,7)$ & $(2 / 3,1 / 3,1 / 2)(5 / 3,1 / 3,-3 / 2)$ & 5.971 & 6 & 0.004 \\
$J(2,8)$ & $(2 / 3,1 / 3,1 / 2)(2 / 3,-5 / 3,-1 / 2)$ & 6.105 & 6 & -0.003 \\
$J(2,9)$ & $(2 / 3,1 / 3,1 / 2)(-1 / 3,5 / 3,-3 / 2)$ & 7.125 & 6 & -0.008 \\
$J(2,10)$ & $(2 / 3,1 / 3,1 / 2)(5 / 3,-5 / 3,1 / 2)$ & 7.275 & 6 & -0.001 \\
$J(2,11)$ & $(2 / 3,1 / 3,1 / 2)(2 / 3,-5 / 3,-3 / 2)$ & 7.638 & 6 & 0.002 \\
$J(2,12)$ & $(2 / 3,1 / 3,1 / 2)(-4 / 3,4 / 3,-1 / 2)$ & 7.743 & 12 & 0.003 \\
$J(2,13)$ & $(2 / 3,1 / 3,1 / 2)(2 / 3,1 / 3,-5 / 2)$ & 7.950 & 1 & 0.000 \\
$J(2,14)$ & & & & 0.008 \\
\hline \hline
\end{tabular}

$\delta \mathrm{VN}_{1-x}$ phase. Other choices considered for nitrogenvacancy sites were found to increase the formation enthalpy. For the first metastable phase, $\mathrm{V}_{16} \mathrm{~N}$, it is seen from Table II that the calculated equilibrium lattice parameters are in good agreement with experimental values. Although the variation in b lattice constant by $2.7 \%$ from experiment is on the upper limit of DFT accuracy, it may be noted that the structure used in the calculation is built from an ordering of $\mathrm{N}$ atoms in the $\mathrm{V}$ sublattice based on experimental information where the lattice parameter is known to show variation with composition of the alloy. The formation enthalpy is calculated to be $-156 \mathrm{meV} /$ atom. For the second metastable phase, $\mathrm{V}_{9} \mathrm{~N}_{2}$ (or $\mathrm{V}_{9} \mathrm{~N}_{1}$ ), a set of three calculations were carried out corresponding to different $\mathrm{N}$ occupancy in the structure as described in Sec. I. Table II lists corresponding lattice parameters and formation enthalpies. The $\mathrm{V}_{54} \mathrm{~N}_{6}-\mathrm{V}_{9} \mathrm{~N}_{1}$ (Oct1) and $\mathrm{V}_{54} \mathrm{~N}_{12}-\mathrm{V}_{9} \mathrm{~N}_{2}$ (Tet) involves nitrogen occupancy in the usual octahedral and tetrahedral interstitial sites of bcc lattice, respectively. The $\mathrm{V}_{54} \mathrm{~N}_{12}-\mathrm{V}_{9} \mathrm{~N}_{2}$ (Oct2) corresponds to nitrogen atoms located in the other octahedral sites specific to the space group of the phase. It is evident that the calculated equilibrium lattice parameter of $\mathrm{V}_{54} \mathrm{~N}_{6}-\mathrm{V}_{9} \mathrm{~N}_{1}$ (Oct1) is in close agreement with the experimental value. For other choices, our calculated lattice parameters are larger by $2.5-3 \%$ of the experimental values. Further, the calculated formation enthalpy is significantly lower for $\mathrm{V}_{54} \mathrm{~N}_{6}-\mathrm{V}_{9} \mathrm{~N}_{1}$ (Oct1) than that of $\mathrm{V}_{54} \mathrm{~N}_{12}-\mathrm{V}_{9} \mathrm{~N}_{2}$ (Oct2). The formation enthalpy of $\mathrm{V}_{54} \mathrm{~N}_{12}-\mathrm{V}_{9} \mathrm{~N}_{2}$ (Tet) is comparable to that of $\mathrm{V}_{54} \mathrm{~N}_{6}-\mathrm{V}_{9} \mathrm{~N}_{1}$ (Oct1). These formation enthalpies thus show a preference to $\mathrm{V}_{9} \mathrm{~N}_{1}$ over $\mathrm{V}_{9} \mathrm{~N}_{2}$. Between the two choices of $\mathrm{V}_{9} \mathrm{~N}_{2}$, the one with nitrogen atoms located in the regular tetrahedral sites is the more likely possibility. The lowformation enthalpy of $\mathrm{V}_{9} \mathrm{~N}_{2}$ (Tet), comparable to the minimum energy $\mathrm{V}_{9} \mathrm{~N}_{1}$ (Oct1), is in accordance with the fact that the $\mathrm{V}_{9} \mathrm{~N}_{2}$ (Tet) is analogous to $\mathrm{Ta}_{9} \mathrm{~N}_{2}$ phase with $\mathrm{N}$ atoms in tetrahedral sites. Such an isomorphism is not surprising as V and $\mathrm{Ta}$ are from the same group in the periodic table. Thus, our DFT-calculated formation enthalpies distinguish the three possible configurations considered for the second metastable phase, which was very difficult to resolve by electrondiffraction study. ${ }^{10}$

For the third metastable phase, $\mathrm{V}_{8} \mathrm{~N}$, our calculated lattice parameters (Table II) are in good agreement with the experimental value. We further observe that the formation enthalpy of the metastable phases is an order of magnitude higher compared to that of the equilibrium phases.

The results of supercell calculation for pseudobinary formation enthalpy of $\mathrm{VN}_{1-x} \square_{x}$ are shown in Fig. 2. Formation enthalpies of NaCl- and WC-type $\mathrm{VN}_{1-x} \square_{x}$ are plotted with respect $\mathrm{VN}$ and $\mathrm{V} \square$ for various values of $x$. It is evident that nitrogen vacancies stabilize $\mathrm{NaCl}$ phase rather than $\mathrm{WC}$ phase establishing the calculated phase stability in agreement with experiments. We complement these results with cluster expansion study in the next section. In Fig. 3, we have presented our calculated formation enthalpy of the various phases of stable and metastable vanadium nitrides, compared with experiments where available. The formation enthalpies of low-energy phases are seen to decrease in the same order as in the aging sequence. Figure 3 is actually an updated version presented in a previous work [Ravi (Ref. 13)] and the reader may refer to this paper for details on the phases for which more information is required. 

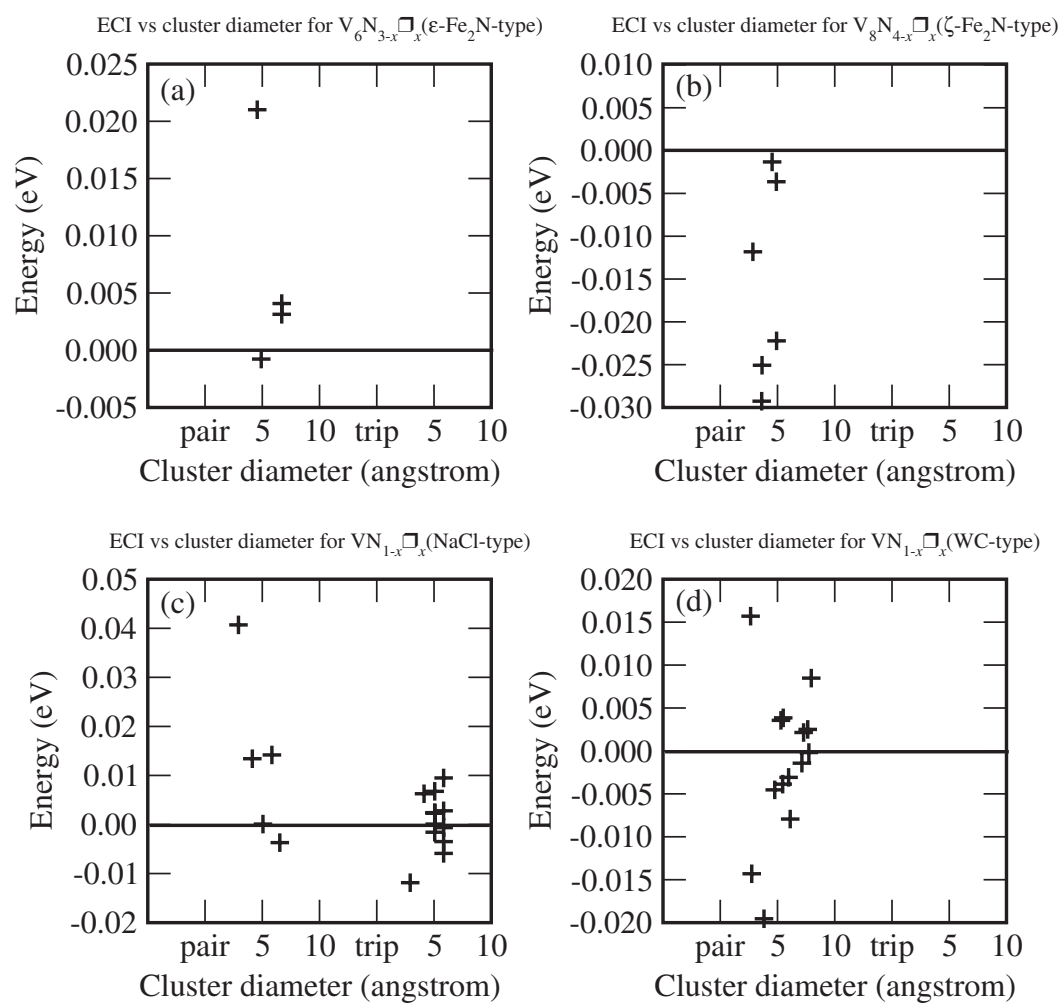

FIG. 5. Calculated ECI versus cluster diameter. $X$ axis shows cluster diameters, first for pair clusters beginning with label "pair," next for triplet clusters, and so on. Cluster diameter is defined as the length of the longest pair contained in the cluster. Although triplet clusters are present only in plot (c), the $X$-axis scale is maintained same in all the plots for the sake of easy comparison. It is evident that the magnitudes of the ECI's generally decrease with distance and with cluster size. We see that for $\mathrm{V}_{2} \mathrm{~N}_{1-x} \square_{x}$, convergence of the cluster expansion is fairly fast, requiring a maximum of six pair terms. In the case of $\mathrm{VN}_{1-x} \square_{x}$, convergence is relatively slow. For $\mathrm{NaCl}$ phase, interactions up to fifth neighbor pairs and 12 three-body terms are required to obtain the desired CV score. Cluster expansion of WC phase includes interactions up to 14 th neighbor pairs.

\section{B. Cluster expansion and phase stability}

In this section, we present the results of cluster expansion Monte Carlo simulation for the phase stability and thermodynamic properties of $\mathrm{V}_{2} \mathrm{~N}_{1-x} \square_{x}$ and $\mathrm{VN}_{1-x} \square_{x}$. For $\mathrm{V}_{2} \mathrm{~N}_{1-x} \square_{x}$, two cluster expansions were constructed with $\epsilon-\mathrm{Fe}_{2} \mathrm{~N}$ and $\zeta-\mathrm{Fe}_{2} \mathrm{~N}$ structures as parent lattices. For $\mathrm{VN}_{1-x} \square_{x}$, the cluster expansions were constructed with Na$\mathrm{Cl}(\mathrm{B} 1)$ and $\mathrm{WC}\left(\mathrm{B}_{h}\right)$ structures as parent lattices. Results of cluster-expansions and ground-state searches (via direct enumeration) showing the formation energies of $\mathrm{V}_{2} \mathrm{~N}_{1-x} \square_{x}$ and $\mathrm{VN}_{1-x} \square_{x}$ pseudobinary systems are given in Fig. 4 . The ECI obtained from cluster expansions for each of the four parent lattices are give in Tables III-V and are shown in Fig. 5. Unlike ECI's in a binary alloy system, the pair and threebody interaction parameters in a pseudobinary system are considered as pseudopair and pseudo-three-body interaction parameters due to the interactions with the spectator specie. Actually, each ECI in the CE method renormalizes in it all interatomic potentials (two-body, three-body, etc., summed over all interatomic distances) so that even the nearestneighbor-pair ECI of CE contains information from all interatomic interactions.

Therefore the CE method is often referred to as "renormalized interaction" approach. ${ }^{30}$ It is evident that the magnitudes of the ECI's generally decay with distance and with cluster size. In the case of $\mathrm{V}_{2} \mathrm{~N}_{1-x} \square_{x}$, convergence of the cluster expansion is fairly fast requiring a maximum of six pair terms while in the case of $\mathrm{VN}_{1-x} \square_{x}$ convergence is relatively slow. For $\mathrm{NaCl}$ phase, interactions up to fifth neighbor pairs and 12 three-body terms are required to obtain the desired CV score. Cluster expansion of WC phase includes interactions up to 14 th neighbor pairs.

As shown in Fig. 4, the cluster expansions of $\mathrm{V}_{2} \mathrm{~N}_{1-x} \square_{x}$ on the $\epsilon-\mathrm{Fe}_{2} \mathrm{~N}$ parent lattice have predicted several ground states between $\mathrm{V}_{6} \mathrm{~N}_{3} \square_{0}$ and $\mathrm{V}_{6} \mathrm{~N}_{2} \square_{1}$ whereas the cluster expansion with $\zeta-\mathrm{Fe}_{2} \mathrm{~N}$-type phase as parent lattice has predicted no intermediate ground states. Similarly, the cluster expansions of $\mathrm{NaCl}$-type $\mathrm{VN}_{1-x} \square_{x}$ has identified several ground states between $\mathrm{VN}$ and $\mathrm{V} \square$ and no intermediate ground-states for WC phase. These cluster expansion results show that nitrogen vacancies lower the formation energy of $\epsilon$-Fe ${ }_{2} \mathrm{~N}$-type $\mathrm{V}_{2} \mathrm{~N}_{1-x} \square_{x}$ and NaCl-type $\mathrm{VN}_{1-x} \square_{x}$ compared to $\zeta-\mathrm{Fe}_{2} \mathrm{~N}$ - and WC-type phases, respectively.

Similar to the cluster-expansion results, our supercell calculation of phase stability of $\mathrm{VN}_{1-x} \square_{x}$ has shown that nitrogen vacancies stabilize $\mathrm{NaCl}$ phase rather than $\mathrm{WC}$ phase. Generally, the nitrogen vacancies in the substoichiometric $\mathrm{VN}_{1-x} \square_{x}$ phase are disordered. Although the supercell calculations ignore the disorder, it is apparent that they have captured the energetic effect of nitrogen vacancies correctly. Therefore, we examine the electronic density of states (DOS) 

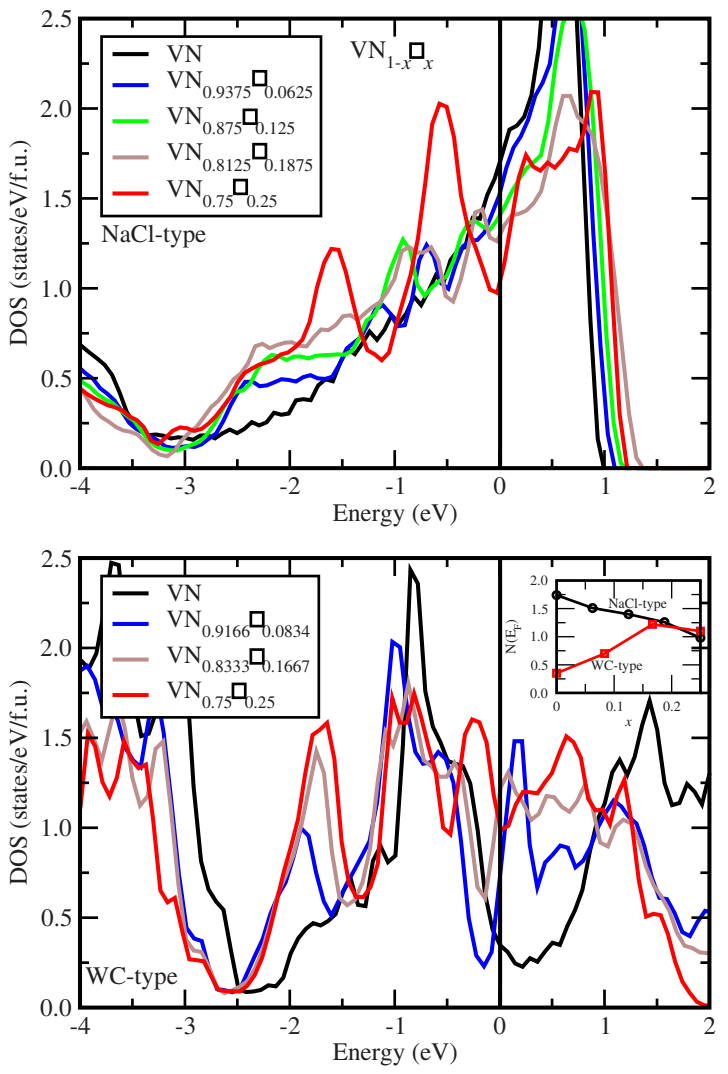

FIG. 6. (Color online) Electronic density of states of $\mathrm{VN}_{1-x} \square_{x}$ as a function of nitrogen-vacancy concentration between $\mathrm{NaCl}$ and WC phases. In both the phases, additional peaks appear in the DOS below Fermi level due to nitrogen vacancies. On the other hand, the DOS at Fermi level, $N\left(E_{F}\right)$, decreases with increasing nitrogen vacancy for $\mathrm{NaCl}$ phase while it increases in the WC phase. Inset in the bottom plot shows this variation in $N\left(E_{F}\right)$ between $\mathrm{NaCl}$ and WC phases.

for understanding the different behavior of nitrogen vacancy on the phase stability of $\mathrm{VN}_{1-x} \square_{x}$ between $\mathrm{NaCl}$ and $\mathrm{WC}$ phases.

DOS near the Fermi level is seen to be considerably affected by incorporation of nitrogen vacancies because this is where vacancy states appear and therefore is critical in determining the phase stability. This is shown in Fig. 6. This part of DOS is made predominately of $\mathrm{V}-d$ states mixed with some amount of N-p states. We see from these figures that vacancy-induced states appear as peaks in the DOS of both $\mathrm{NaCl}$ and $\mathrm{WC}$ phases. However, DOS at the Fermi level, $N\left(E_{F}\right)$, is lowered in the $\mathrm{NaCl}$ phase whereas it is seen to increase in the WC phase with increasing concentration of nitrogen vacancies. It can also be seen that, between $\mathrm{NaCl}$ and WC phases, the occupied DOS curve near the Fermi level has different tendency. That is, for a small but equal amount of decrease in energy, $\mathrm{NaCl}$ phase would release more electron states than the WC phase. This tendency of DOS combined with variation in $N\left(E_{F}\right)$ indicates that nitrogen vacancies stabilize $\mathrm{NaCl}$ phase over the WC phase. ${ }^{44,45}$

Hugosson et al. ${ }^{45}$ have studied the phase stability and bonding mechanisms in stoichiometric and substoichiometric molybdenum carbide. This study shows that carbon vacan- cies lower the energy of $\mathrm{NaCl}$ phase and makes the phase more stable. On the other hand, in the WC phase, carbon vacancies increase the energy, making the phase less stable. In the Mo-C system, both $\mathrm{NaCl}$ - and WC-type $\mathrm{MoC}$ phases are observed. $\mathrm{NaCl}$-type $\mathrm{MoC}$ is known to have wide homogeneity range as is NaCl-type $\mathrm{VN}$ while WC-type MoC has small homogeneity range. Our phase stability calculations thus show that VN behaves like MoC. Similarly, in the Fe-N system, $\epsilon-\mathrm{Fe}_{2} \mathrm{~N}_{1-x}$ is known to be stable in the range from $\epsilon-\mathrm{Fe}_{3} \mathrm{~N}$ to $\epsilon-\mathrm{Fe}_{2} \mathrm{~N}$. When $\epsilon-\mathrm{Fe}_{2} \mathrm{~N}$ stoichiometry has to be reached, $\zeta-\mathrm{Fe}_{2} \mathrm{~N}$ phase is known to appear. The $\zeta-\mathrm{Fe}_{2} \mathrm{~N}$ phase is known to have negligible homogeneity range. ${ }^{46}$ Our cluster expansion results show that nitrogen vacancies stabilize $\epsilon-\mathrm{Fe}_{2} \mathrm{~N}$ phase of $\mathrm{V}_{2} \mathrm{~N}_{1-x} \square_{x}$ rather than $\zeta-\mathrm{Fe}_{2} \mathrm{~N}$ phase. Thus, phase stability of $\mathrm{V}_{2} \mathrm{~N}_{1-x} \square_{x}$ has similar behavior as $\mathrm{VN}_{1-x} \square_{x}$ between $\mathrm{NaCl}$ and $\mathrm{WC}$ phases, possibly due to similar stability mechanism.

We have calculated the formation enthalpy of $\epsilon$ - $\mathrm{Fe}_{2} \mathrm{~N}$-type $\mathrm{V}_{2} \mathrm{~N}_{1-x} \square_{x}$ and $\mathrm{NaCl}(\mathrm{B} 1)$-type $\mathrm{VN}_{1-x} \square_{x}$ for the disordered states using the respective cluster expansion Hamiltonian in a semigrand canonical Monte Carlo simulation. The state of thermodynamic equilibrium for these nonstoichiometric nitrides below $300 \mathrm{~K}$ is known to exhibit order. But, a disordered state is realized as the substance is subjected to normal cooling subsequent to high-temperature synthesis and most of the experimental work deals with the disordered phases. ${ }^{11}$ Melting point of both $\mathrm{V}_{2} \mathrm{~N}_{1-x} \square_{x}$ and $\mathrm{VN}_{1-x} \square_{x}$ are known to be well above $2000 \mathrm{~K}$. Experimental formation enthalpy of $\mathrm{VN}_{1-x} \square_{x}$ is known for $1700 \mathrm{~K}$. In the Monte Carlo simulations, we compute the formation enthalpy of the disordered phases at $T=2000 \mathrm{~K}$. For $\epsilon$-Fe ${ }_{2} \mathrm{~N}$-type $\mathrm{V}_{2} \mathrm{~N}_{1-x} \square_{x}$, our MC simulation used a $22 \times 22 \times 21$ supercell with $60984 \mathrm{~V}$ sites and $30492 \mathrm{~N}$ sites making up a total of 91476 sites. Onethird of the $30492 \mathrm{~N}$ sites, i.e., 10164 , are active sites for sampling with $\square / \mathrm{N}$. For NaCl-type phase, a $22 \times 22 \times 22$ supercell was used. This cell had a total of 21296 sites of which 10648 are active sites occupied by $\mathrm{N} / \square$. For $\zeta-\mathrm{Fe}_{2} \mathrm{~N}-$ and WC-type phases, our Monte Carlo simulations find the disordered state to be thermodynamically unstable for $T$ $\leq 2000 \mathrm{~K}$. This is verified for several combinations of chemical potentials and moderate precision on the average concentration of the alloy. Therefore, Bragg-Williams approximation is employed for computing the formation enthalpy of these phases. The results are shown in Fig. 7.

For $\mathrm{V}_{2} \mathrm{~N}_{1-x} \square_{x}$, measured values of formation enthalpy are available only for values of $x$ close to 0 . The plots in Fig. 7 show that both $\epsilon$ - $\mathrm{Fe}_{2} \mathrm{~N}$ and $\zeta-\mathrm{Fe}_{2} \mathrm{~N}$ phases have approximately same formation enthalpies for low nitrogen-vacancy concentrations. However, with increasing nitrogen vacancies, we see that the mixing enthalpy of the $\zeta-\mathrm{Fe}_{2} \mathrm{~N}$ phase shows a negative curvature while that of the $\epsilon-\mathrm{Fe}_{2} \mathrm{~N}$ phase has a positive curvature. This shows that nitrogen vacancies make $\zeta-\mathrm{Fe}_{2} \mathrm{~N}$ phase unstable whereas they make the $\epsilon-\mathrm{Fe}_{2} \mathrm{~N}$ phase more stable. For $\mathrm{VN}_{1-x} \square_{x}$, our calculated formation enthalpy of $\mathrm{NaCl}$ phase is in good agreement with experimental results. ${ }^{12,47}$ Also, the calculated formation enthalpy of $\mathrm{WC}$ phase has lower value than that of the $\mathrm{NaCl}$ phase near the stoichiometric concentration. However, it becomes higher than that of $\mathrm{NaCl}$ phase as nitrogen vacancies are introduced. This result shows that our cluster expansion Monte Carlo 
Formation energy for $\mathrm{V}_{2} \mathrm{~N}_{1-x} \square_{x}(0<x<0.3333)$

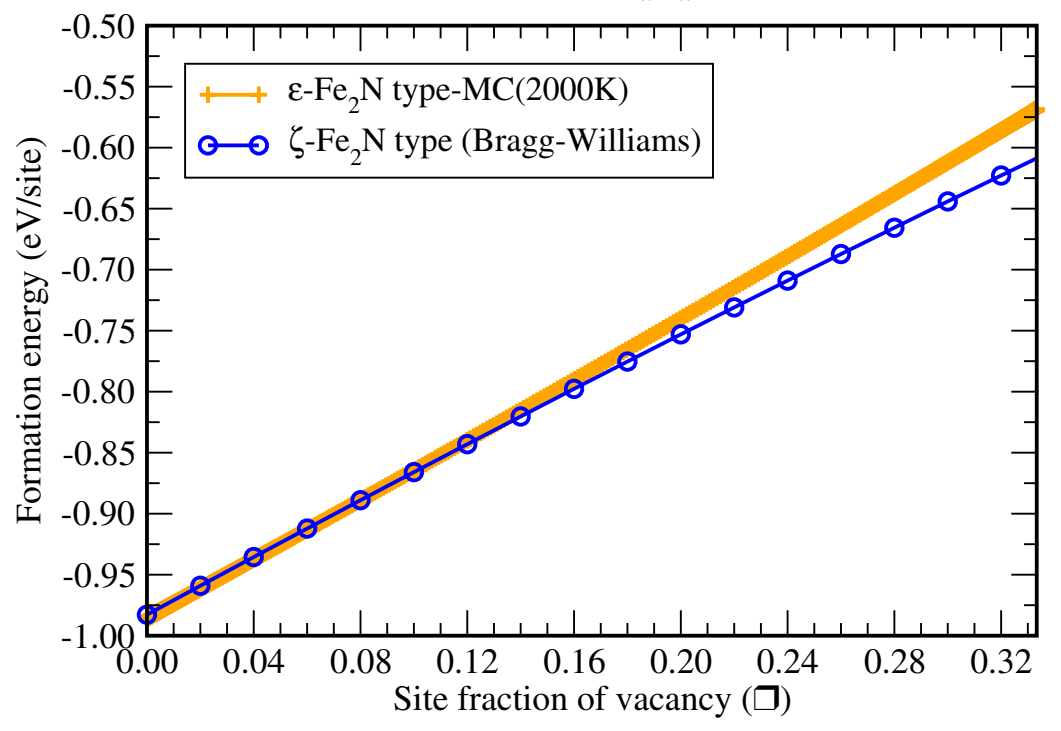

Formation energy for $\mathrm{VN}_{1-x} \square_{x}$

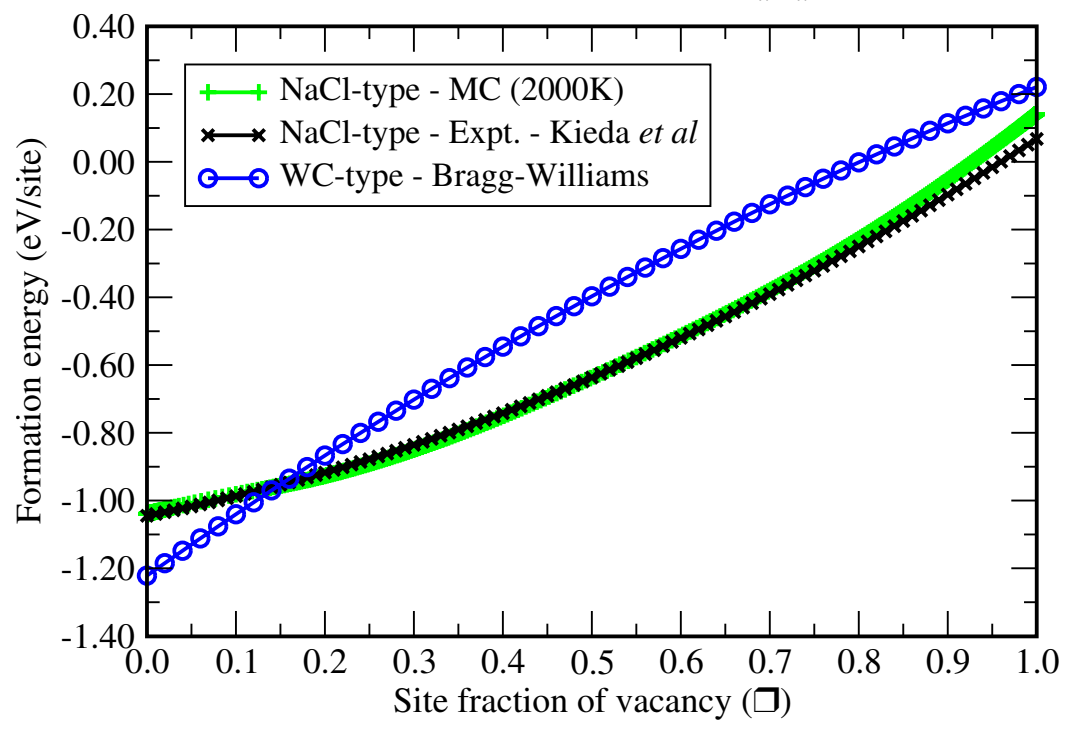

FIG. 7. (Color online) Formation enthalpy of disordered state $\mathrm{V}_{2} \mathrm{~N}_{1-x} \square_{x}$ and $\mathrm{VN}_{1-x} \square_{x}$ compared with the available experimental data. Top plot shows the formation enthalpy of $\mathrm{V}_{2} \mathrm{~N}_{1-x} \square_{x}$ as a function of nitrogen-vacancy concentration for the $\epsilon$ - $\mathrm{Fe}_{2} \mathrm{~N}$ and $\zeta-\mathrm{Fe}_{2} \mathrm{~N}$ phases. In this plot we show formation energy for nitrogen-vacancy concentrations of up to $x=0.3333 \%$ as this is the range for which the cluster expansion was constructed to be accurate. Bottom plot shows the formation enthalpy of $\mathrm{VN}_{1-x} \square_{x}$ for the $\mathrm{NaCl}(\mathrm{B} 1)$ and $\mathrm{WC}\left(\mathrm{B}_{h}\right)$ phases. Melting point of both $\mathrm{V}_{2} \mathrm{~N}_{1-x} \square_{x}$ and $\mathrm{VN}_{1-x} \square_{x}$ are known to be well above $2000 \mathrm{~K}$. Experimental formation enthalpy of $\mathrm{VN}_{1-x} \square_{x}$ is known for $1700 \mathrm{~K}$ [Kieda et al. (Ref. 47)]. In the Monte Carlo simulation, we fixed $T=2000 \mathrm{~K}$. For $\zeta-\mathrm{Fe}_{2} \mathrm{~N}$ and $\mathrm{WC}\left(\mathrm{B}_{h}\right)$ phases, the Monte Carlo simulations finds the disordered state to be thermodynamically unstable for $T \leq 2000 \mathrm{~K}$. However, for these phases, we show the formation enthalpies obtained from Bragg-Williams approximation.

simulations combined with DFT calculations enables calculation of formation enthalpy of random phase in close agreement with experiments.

\section{SUMMARY AND CONCLUSIONS}

We have studied the phase stability of stable and metastable vanadium nitrides using density functional theory based total-energy calculations combined with cluster expansion Monte Carlo simulation and supercell methods. Com- puted formation enthalpies of the various stable and metastable vanadium nitrides considering the available structural models are found to be in close agreement with experimental values where available. The formation enthalpies of all the phases, seen in aging experiments, have been computed and we find them to follow the same sequence in which they appear in the experiments. For one of the metastable phases, $\mathrm{V}_{9} \mathrm{~N}_{2}$ (or $\mathrm{V}_{9} \mathrm{~N}_{1}$ ), experiments indicate three possible ordering of nitrogen atoms but it was difficult to resolve among the three by electron-diffraction studies. Our DFT-based 
study of energetics resolves this and shows that $\mathrm{V}_{9} \mathrm{~N}_{1}$ with $\mathrm{N}$ placed in the usual octahedral interstitial sites has lowest energy compared to $\mathrm{V}_{9} \mathrm{~N}_{2}$. Between $\mathrm{V}_{9} \mathrm{~N}_{2}$ (Tet) and $\mathrm{V}_{9} \mathrm{~N}_{2}$ (Oct2), first one with $\mathrm{N}$ atoms in tetrahedral sites is seen to have lower energy. It is known that DFT calculation shows stoichiometric $\mathrm{V}_{2} \mathrm{~N}$ to be polymorphic in $\epsilon-\mathrm{Fe}_{2} \mathrm{~N}$ and $\zeta-\mathrm{Fe}_{2} \mathrm{~N}$ structures within a few meV and VN more stable in $\mathrm{WC}\left(\mathrm{B}_{h}\right)$ phase than experimental $\mathrm{NaCl}(\mathrm{B} 1)$ structure. The results of the present study demonstrate that the $\mathrm{N}$ vacancies in $\mathrm{VN}$ render the observed $\mathrm{NaCl}$ structure stable though stoichiometric VN has WC structure. Similarly, the $\epsilon-\mathrm{Fe}_{2} \mathrm{~N}$ structure of $\mathrm{V}_{2} \mathrm{~N}$ is shown to be more stable compared to the stoichiometric $\zeta-\mathrm{Fe}_{2} \mathrm{~N}$ structure.

Analysis of supercell calculated electronic DOS of $\mathrm{VN}_{1-x} \square_{x}$ with varying $x$, in $\mathrm{NaCl}$ and $\mathrm{WC}$ phases, shows that, in both the phases, the DOS peak below the Fermi level is shifted toward lower energy, with appearance of additional states due to nitrogen vacancies. On the other hand, the nitrogen vacancies increase the DOS at Fermi level in WC phase, whereas decrease the DOS in $\mathrm{NaCl}$ phase, hence we identify this to be a stability mechanism. Comparing V-N system to $\mathrm{Fe}-\mathrm{N}$ system, $\epsilon$ - $\mathrm{Fe}_{2} \mathrm{~N}_{1-x}$ is experimentally found to be more stable than $\epsilon-\mathrm{Fe}_{2} \mathrm{~N}$. On decreasing $x$ from 0.33 to 0 , the $\zeta-\mathrm{Fe}_{2} \mathrm{~N}$ phase appears as the stable phase over the $\epsilon-\mathrm{Fe}_{2} \mathrm{~N}$ (Ref. 46). The $\zeta-\mathrm{Fe}_{2} \mathrm{~N}$ phase is known to have a narrow homogeneity range. Our cluster expansion results confirm that nitrogen vacancies stabilize $\epsilon$ - $\mathrm{Fe}_{2} \mathrm{~N}$ phase of $\mathrm{V}_{2} \mathrm{~N}_{1-x} \square_{x}$ rather than $\zeta-\mathrm{Fe}_{2} \mathrm{~N}$ phase. In this way, phase stability of $\mathrm{V}_{2} \mathrm{~N}_{1-x} \square_{x}$ has similar behavior as $\mathrm{VN}_{1-x} \square_{x}$ between $\mathrm{NaCl}$ and WC phases, possibly due to similar stability mechanism. In addition to the $T=0 \mathrm{~K}$ calculations, Monte Carlo simulations were used for computing the finite-temperatureformation enthalpies of these nitrides as a function of nitrogen-vacancy concentration and found close agreement for $\mathrm{NaCl}(\mathrm{B} 1)$ phase of $\mathrm{VN}_{1-x} \square_{x}$, for which measured values are available. This strengthens the efficacy of cluster expansion Monte Carlo methods combined with DFT calculations for studying phase equilibrium in transition-metal nitrides exhibiting characteristic nonstoichiometric interstitial compound phases.

\section{ACKNOWLEDGMENTS}

One of the authors, C. Ravi, thanks B. K. Panigrahi for the discussion and support for the work. Axel van de Walle acknowledges that this research was supported by the U.S. National Science Foundation through TeraGrid resources provided by NCSA under Grant No. DMR050013N, through the U.S. Department of Energy, National Energy Research Initiative Consortium (NERI-C) under Grant No. DE-FG0707 ID14893. *ravic@igcar.gov.in

${ }^{1}$ K. Maile and E. Roos, in Frontiers in the Design of Materials, Series in Metallurgy and Materials Science, edited by Baldev Raj, S. Ranganathan, S. L. Mannan, K. Bhanu Sankara Rao, M. D. Mathew, and P. Shankar, (Universities Press, Hyderabad, 2007), p.141.

${ }^{2}$ F. Abe, Sci. Technol. Adv. Mater. 9, 013002 (2008).

${ }^{3}$ P. Lazar, R. Podloucky, E. Kozeschnik, and J. Redinger, Phys. Rev. B 78, 134202 (2008).

${ }^{4}$ H. Holleck, J. Vac. Sci. Technol. A 4, 2661 (1986).

${ }^{5}$ J.-G. Choi and M.-K. Jung, J. Ind. Eng. Chem. (Seoul, Repub. Korea) 3, 93 (1997).

${ }^{6}$ S.-H. Jhi, J. Ihm, S. G. Louie, and M. L. Cohen, Nature (London) 399, 132 (1999).

${ }^{7}$ W. H. Hugosson, U. Jansson, B. Johansson, and O. Eriksson, Science 293, 2434 (2001)

${ }^{8}$ T. Joelsson, L. Hultman, H. W. Hugosson, and J. M. MolinaAldareguia, Appl. Phys. Lett. 86, 131922 (2005).

${ }^{9}$ R. de Paiva, R. A. Nogueira, and J. L. A. Alves, Phys. Rev. B 75, 085105 (2007).

${ }^{10}$ O. N. Carlson, J. F. Smith, and R. H. Nafziger, Metall. Trans. A 17, 1647 (1986).

${ }^{11}$ A. I. Gusev, Phys. Status Solidi B 163, 17 (1991).

${ }^{12}$ A. I. Gusev, Phys. Status Solidi B 209, 267 (1998).

${ }^{13}$ C. Ravi, CALPHAD:Comput. Coupling Phase Diagrams Thermochem. 33, 469 (2009).

${ }^{14}$ T. Onozuka, J. Appl. Crystallogr. 11, 132 (1978).

${ }^{15}$ D. Potter and G. Altstetter, Acta Metall. 19, 881 (1971).

${ }^{16}$ D. I. Potter, H. D. Epstein, and B. M. Goldstein, Metall. Trans.
5, 2075 (1974).

${ }^{17}$ L. N. Galkin, V. V. Vavilova, and L. Ye Fykin, Phys. Met. Metallogr. 45, 67 (1979).

${ }^{18}$ P. D. Tepesch, G. D. Garbulsky, and G. Ceder, Phys. Rev. Lett. 74, 2272 (1995)

${ }^{19}$ G. Ceder, A. van der Ven, C. Marianetti, and D. Morgan, Modell. Simul. Mater. Sci. Eng. 8, 311 (2000).

${ }^{20} \mathrm{G}$. Ghosh, A. van de Walle, and M. Asta, J. Phase Equilib. Diffus. 28, 9 (2007).

${ }^{21}$ J. W. D. Connolly and A. R. Williams, Phys. Rev. B 27, 5169 (1983).

${ }^{22}$ J. M. Sanchez, F. Ducastelle, and D. Gratias, Physica A 128, 334 (1984).

${ }^{23}$ D. de Fontaine, in Electronic Band Structure and Its Applications, Lecture Notes in Physics Vol. 283, edited by M. Yussouff (Springer-Verlag, Berlin, 1987), p. 410.

${ }^{24}$ J. M. Sanchez, J. P. Stark, and V. L. Moruzzi, Phys. Rev. B 44, 5411 (1991).

${ }^{25}$ Z. W. Lu, S.-H. Wei, A. Zunger, S. Frota-Pessoa, and L. G. Ferreira, Phys. Rev. B 44, 512 (1991).

${ }^{26}$ G. Ceder, Comput. Mater. Sci. 1, 144 (1993).

${ }^{27}$ M. Asta, R. McCormack, and D. de Fontaine, Phys. Rev. B 48, 748 (1993).

${ }^{28}$ V. Ozoliņš and J. Haglund, Phys. Rev. B 48, 5069 (1993).

${ }^{29}$ C. Wolverton and A. Zunger, Phys. Rev. B 50, 10548 (1994).

${ }^{30}$ A. Zunger, in Statics and Dynamics of Alloy Phase Transformations, edited by P. E. A. Turchi and A. Gonis (Plenum, New York, 1994), p. 361.

${ }^{31}$ P. A. Korzhavyi, L. V. Pourovskii, H. W. Hugosson, A. V. Ruban, 
and B. Johansson, Phys. Rev. Lett. 88, 015505 (2001).

${ }^{32}$ A. van de Walle and G. Ceder, J. Phase Equilib. 23, 348 (2002).

${ }^{33}$ G. L. W. Hart, B. M. Klein, and S. Begay, in Complex Inorganic Solids, edited by P. E. A. Turchi, A. Gonis, K. Rajan, and A. Meike, (Springer, New York, 2005), p. 99.

${ }^{34}$ A. V. Ruban and I. A. Abrikosov, Rep. Prog. Phys. 71, 046501 (2008).

${ }^{35}$ L. G. Ferreira, S.-H. Wei, and A. Zunger, Int. J. Supercomput. Appl. 5, 34 (1991).

${ }^{36}$ A. van de Walle and M. Asta, Modell. Simul. Mater. Sci. Eng. 10, 521 (2002).

${ }^{37}$ G. Kresse and J. Hafner, Phys. Rev. B 47, 558 (1993).

${ }^{38}$ G. Kresse and J. Furthmuller, Phys. Rev. B 54, 11169 (1996).
${ }^{39}$ D. Vanderbilt, Phys. Rev. B 41, 7892 (1990).

${ }^{40}$ J. P. Perdew, in Electronic Structure of Solids, edited by P. Ziesche and H. Eschrig (Akademie, Berlin, 1991), Vol. 11.

${ }^{41}$ M. Methfessel and A. T. Paxton, Phys. Rev. B 40, 3616 (1989).

${ }^{42}$ H. J. Monkhorst and J. D. Pack, Phys. Rev. B 13, 5188 (1976).

${ }^{43}$ ATAT manual, http://www.its.caltech.edu/ avdw/atat

${ }^{44}$ D. L. Price and B. R. Cooper, Phys. Rev. B 39, 4945 (1989).

${ }^{45}$ H. W. Hugosson, O. Eriksson, L. Nordstrom, U. Jansson, L. Fast, A. Delin, J. M. Wills, and B. Johansson, J. Appl. Phys. 86, 3758 (1999).

${ }^{46}$ D. H. Jack and K. H. Jack, Mater. Sci. Eng. 11, 1 (1973).

${ }^{47}$ N. Kieda, N. Mizutani, and M. Kato, J. Less-Common Met. 144, 293 (1988). 